\title{
Influence of amino acids on gastric adaptive relaxation (accommodation) in rats as evaluated with a barostat
}

\author{
Masayuki Uchida and Chizuru Iwamoto \\ Food Science Research Laboratories, Research and Development Division, Meiji Co., Ltd., \\ Kanagawa, Japan
}

\begin{abstract}
Aim: The present study aimed to evaluate the effects of selected straight alkyl chain, hydroxylated chain and branched chain amino acids on gastric adaptive relaxation, as these have previously been shown to have differing effects on gastric emptying. Materials and Methods: Gastric adaptive relaxation was evaluated using a barostat in rats under urethane anesthesia. The pressure within the balloon, introduced from the mouth to the stomach, was changed stepwise from 1 to $8 \mathrm{mmHg}$. The increased volume just after the increase of balloon pressure was defined as distension-induced gastric adaptive relaxation (accommodation). Amino acids were administered orally or intravenously. Results: As compared with control rats administered with distilled water, those rats that were orally administered amino acids having straight alkyl chain and extra hydroxylated alkyl chain, such as glycine and L-serine, had significantly enhanced gastric adaptive relaxation, but administration of L-alanine and L-threonine did not. Branched chain amino acids, such as L-isoleucine, L-leucine and L-valine, also did not significantly influence gastric adaptive relaxation. Glycine and L-serine showed the same efficacy when administered intravenously. Conclusion: Among the amino acids evaluated in the present study, glycine and L-serine significantly enhanced gastric adaptive relaxation, suggesting that short alkyl chain amino acids may enhance gastric adaptive relaxation as compared with the other amino acids. These findings may suggest that glycine and L-serine would be useful in the therapy of functional dyspepsia, especially for early satiety, because the dysfunction of adaptive relaxation is one of the causes of early satiety.
\end{abstract}

Key words: Amino acid, Barostat study in rats, Gastric adaptive relaxation, gastric emptying

Corresponding author: Masayuki Uchida, Food Science Research Laboratories, Research and Development Division, Meiji Co., Ltd. 540 Naruda, Odawara, Kanagawa, 250-0862 Japan

Phone: +81-465-37-3664 Fax: +81-465-37-3638 e-mail: masayuki.uchida@maiji.com

(C2016 The Japan Society of Smooth Muscle Research 


\section{Introduction}

In 2013, Jordi et al. (1) reported that L-arginine, L-lysine and L-glutamic acid inhibited food intake via the area postrema or vagal afferents. Carney et al. (2) found that a sense of fullness was greater after L-tryptophan than after ingestion of D-tryptophan, suggesting a delay in gastric emptying. We also observed a significant delay in gastric emptying with treatment with L-tryptophan using the ${ }^{13} \mathrm{C}$-breath test (3). In the previous paper, we reported that amino acids having either an alkyl chain with a hydroxyl group, or a branched chain, differently inhibited and/or delayed gastric emptying, by analyzing the change of expired ${ }^{13} \mathrm{CO}_{2}$ air and the $\mathrm{Cmax}$, Tmax and $\mathrm{AUC}_{120 \text { min }}$ values as evaluated by the noninvasive breath test using $\left[1-{ }^{13} \mathrm{C}\right]$ acetic acid in conscious rats (4). However, the effects of these amino acids on gastric adaptive relaxation have not been clarified in relation to the structure of different amino acids.

In basic studies using experimental animals, many reports have investigated changes in intra-gastric pressure by surgically inserting a pressure transducer into the stomach of rats or mice (5-8). However, such surgical intervention would affect gastric physiological function in an in vivo study. We recently improved the method to evaluate gastric adaptive relaxation in rats without surgery (9).

Therefore, in the present study we evaluated the effects of amino acids having either a straight alkyl chain (glycine and L-alanine), an extra hydroxylated alkyl chain ( $\mathrm{L}^{-}$-serine and $\mathrm{L}^{-}$-threonine) or a branched chain (L-isoleucine, L-leucine and L-valine) on gastric adaptive relaxation using our improved method (9).

\section{Materials and Methods}

The following animal studies were performed in accordance with the Guiding Principles for the Care and Use of Laboratory Animals approved by Meiji Co., Ltd.

\section{Animals}

Male Sprague-Dawley rats weighing about $200 \mathrm{~g}$ were purchased from SLC (Shizuoka, Japan) and housed for 1 week prior to the commencement of the experiments under a constant temperature of $21 \pm 2$ degree centigrade, with a humidity of $55 \pm 15 \%$ and exposed to a 12-hour light/dark cycle. The rats were fasted in mesh cages for $18 \mathrm{hrs}$ before each experiment in order to prevent coprophagy, but were allowed free access to drinking water during this period.

\section{Barostat study}

Gastric adaptive relaxation was evaluated by the method previously reported by us (9). Rats were anesthetized with urethane $(1.2 \mathrm{~g} / \mathrm{kg}$, i.p.). In this study we used a modified balloon, by removing the tube within the balloon to improve the fitting of the balloon to the fundus (Fig. 1A right). A polyvinyl tube with an adherent polyethylene balloon (maximum volume $7 \mathrm{ml} ; 3 \mathrm{~cm}$ maximum diameter) was introduced from the mouth to the stomach (Fig. 1A left). Five $\mathrm{ml}$ of air was injected into the balloon via a second balloon tube under the closed first balloon tube used for fitting the balloon to the stomach as shown in Fig. 1A right. The second balloon tube was immediately opened to the air. After 5 min recovery time, the tube of the balloon was connected to the barostat (Barostat Distender IIR, G\&J Electronics, Toronto Canada). The pressure of the balloon was changed stepwise, from 1 , to 2 , then 4 and $8 \mathrm{mmHg}$, at $1 \mathrm{~min}$ intervals. The volume of the balloon increased correspondingly. When the pressure was raised the balloon inflated quickly and then the volume gradually increased reaching a plateau within one min. The amplitude of this gradually increased component was used 
A
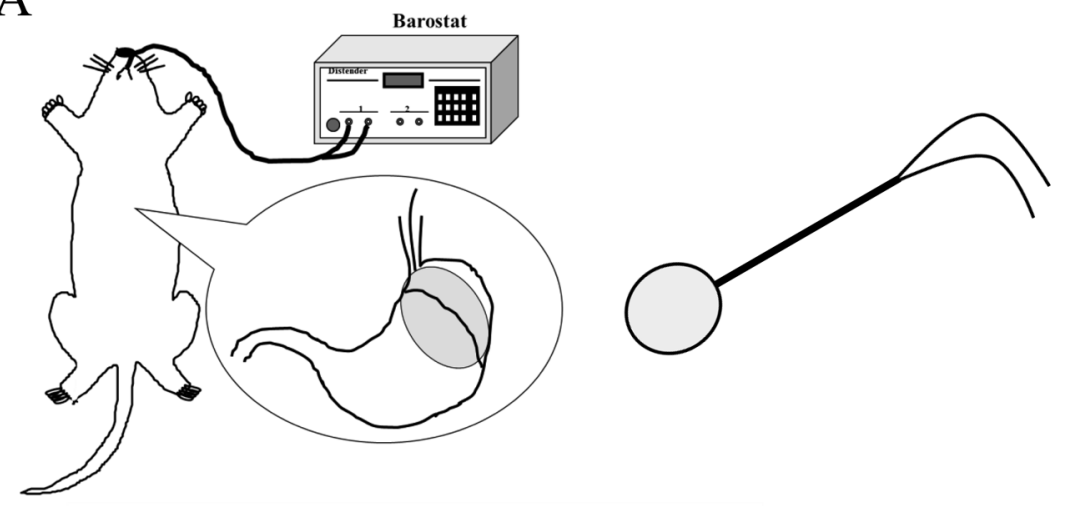

B
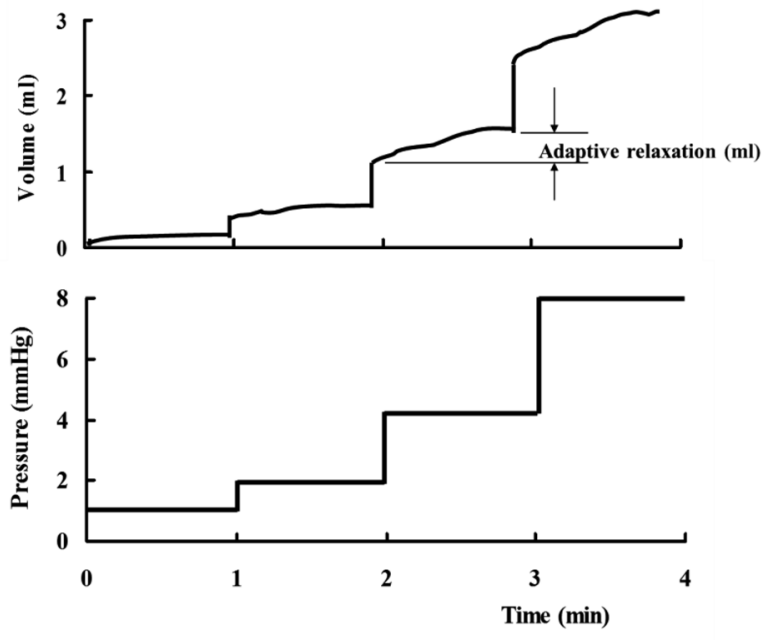

C

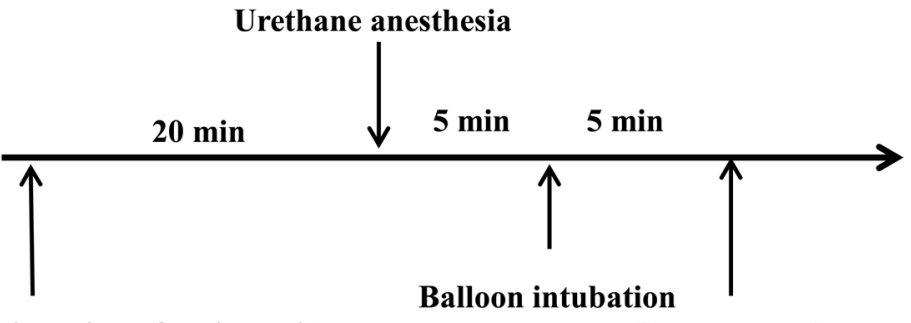

Oral administration of amino acid or distilled water

Barostat study

Fig. 1. Schematic representation of the experimental setting, the changes of the balloon pressure and gastric adaptive relaxation, and time schedule of the experiment. Note the following: A: A slightly improved balloon with the tube within the balloon removed was used in this study so that the balloon fitted more closely within the fundus. The balloon was introduced from the mouth to the stomach in anesthetized rats without surgical operation and set at the fundus. The balloon has two tubes connecting to the barostat. B: The balloon volume increased gradually just after the increment of the balloon pressure, and allowed to plateau for about $1 \mathrm{~min}$. We defined this increased volume as the gastric adaptive relaxation. C: Amino acids were administered orally $30 \mathrm{~min}$ before the barostat study. Twenty min after the amino acids administration, rats were anesthetized by intraperitoneal administration of urethane $(1.2 \mathrm{~g} / \mathrm{kg})$. The balloon was introduced from the mouth to the stomach $5 \mathrm{~min}$ after anesthesia and the barostat study performed 5 min later. 
as a measure of the adaptive relaxation (see Fig. 1B).

Following the evaluation of gastric adaptive relaxation, each rat was dissected and the position of the balloon visually checked to determine correct positioning in the fundus. If the balloon was not in the right position, the data from that animal was excluded from the results.

\section{Effects of orally administered amino acids on the gastric adaptive relaxation}

In the present study we used amino acids having either a straight alkyl chain, such as glycine and L-alanine, an extra hydroxylated alkyl chain, such as L-serine and L-threonine, or a branched chain, such as L-isoleucine, L-leucine and L-valine. After fasting, $1 \mathrm{~g} / \mathrm{kg}$ of amino acid dissolved or suspended in distilled water for injection was administered orally in a volume of $5 \mathrm{ml} / \mathrm{kg}$. In control rats, distilled water for injection was administered instead of the amino acid solution. Barostat study was performed 30 min after administration of the amino acid solution. The time schedule used in the present experiments is shown in detail in Fig. 1C.

The dosage of amino acids ( $1 \mathrm{~g} / \mathrm{kg})$ was identical to that in our earlier study (4) to allow a comparison between the gastric adaptive relaxation and gastric emptying studies.

\section{Effects of intravenously administered glycine and L-serine on gastric adaptive relaxation}

In this study, because orally administered glycine and L-serine showed significant enhancement of gastric adaptive relaxation, we examined the effects of intravenously administered glycine and L-serine.

The pressure of the balloon was changed stepwise, from 1, to 2 and then $4 \mathrm{mmHg}$, at $1 \mathrm{~min}$ intervals. One min after the balloon pressure was changed from 2 to $4 \mathrm{mmHg}$, an amino acid solution was administered intravenously at a dosage of $100 \mathrm{mg} / \mathrm{kg}(2 \mathrm{ml} / \mathrm{kg})$ dissolved in saline. In the control rats, saline was administered instead of amino acid solution. The increased volume of the balloon when it reached a plateau was calculated either 3 min after or within $3 \mathrm{~min}$. The increase in volume was used as a measure of adaptive relaxation $(\mathrm{ml})$.

\section{Agents}

Amino acids used in the present study were purchased from Wako Pure Chemical (Tokyo, Japan). Both distilled water for injection and saline were obtained from Otsuka Pharmaceutical Factory, Inc. (Tokushima, Japan).

\section{Data analysis}

All results are presented as the mean \pm standard error (S.E.). Statistical analysis was performed by Dunnett's multiple comparison test and $P<0.05$ was considered to be significant.

\section{Results}

\section{Effects of orally administered amino acids on the gastric adaptive relaxation}

In the control group, the volume of gastric adaptive relaxation increased with the increment of the balloon pressure as shown in Fig. 2, and a positive correlation was observed between the balloon pressure and the gastric adaptive relaxation. However, significance could not be calculated because with 4 observations, the degrees of freedom was 2 . 


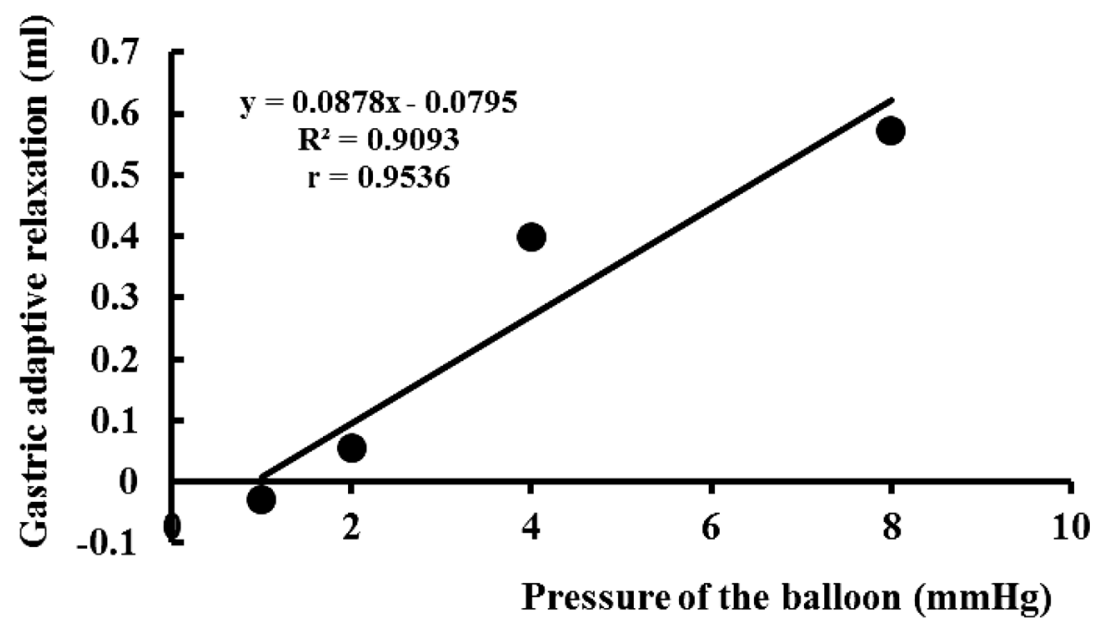

Fig. 2. Correlation between the balloon pressure and changes in the adaptive relaxation in control rats. Adaptive relaxation increased with each increment of the balloon pressure. A positive correlation was observed between the pressure and the adaptive relaxation. Values represent the mean \pm standard error of the mean (SEM) $(n=5)$.

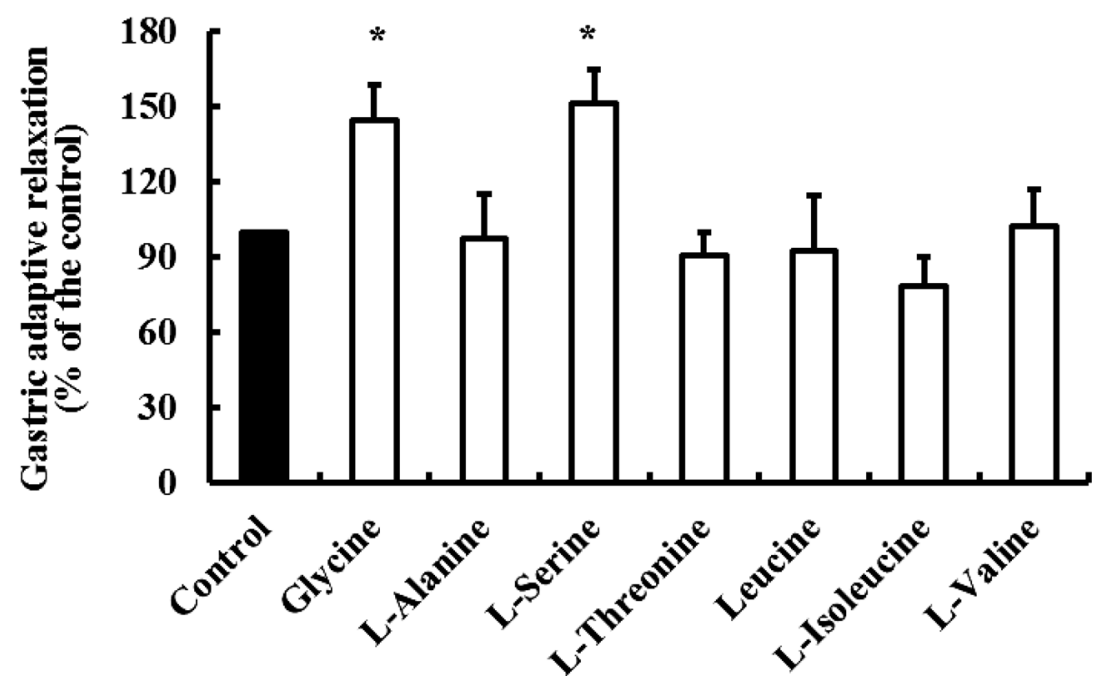

Fig. 3. Effects of orally administered amino acids on gastric adaptive relaxation in rats. Gastric adaptive relaxation was expressed as a percentage of that in control rats. In the control rats, gastric adaptive relaxation was $0.57 \pm 0.11 \mathrm{ml}$ at $8 \mathrm{mmHg}$. Values were expressed as the percentage of the control at $8 \mathrm{mmHg}$, and represent the mean \pm standard error of the mean (SEM) $(\mathrm{n}=5$ or 6$)$. *: Significant difference observed as compared with control $(P<0.05)$.

Adaptive relaxation showed the highest value at $8 \mathrm{mmHg}$. Therefore, we used a pressure of $8 \mathrm{mmHg}$ to evaluate the effects of amino acids hereafter. The effects of amino acids were expressed as a percentage of the control at $8 \mathrm{mmHg}$.

In the control group, the gastric adaptive relaxation was $0.57 \pm 0.11 \mathrm{ml}$ at $8 \mathrm{mmHg}$. The effects of amino acids used were shown in Fig. 3. In the straight alkyl chain and extra hydroxylated alkyl chain amino acids, glycine and L-serine significantly enhanced the gastric adaptive relaxation as compared with the control group $(P<0.05)$, but L-alanine and L-threonine did not. The branched chain amino acids, L-isoleucine and L-leucine and L-valine, also did not significantly influence gastric adaptive relaxation. 


\section{Straight alkyl chain amino acids}<smiles>NCC(=O)O</smiles><smiles>C[C@H](N)C(=O)O</smiles>

\section{Amino acids having extra hydroxylated alkyl chain}<smiles>N[C@@H](CO)C(=O)O</smiles><smiles>C[C@H](O)[C@H](N)C(=O)O</smiles>

\section{Branched chain amino acids}<smiles>CC[C@H](C)[C@H](N)C(=O)O</smiles><smiles>CC(C)[C@@H](N)C(=O)O</smiles>

Fig. 4. Chemical structures of the amino acids and their gastric adaptive relaxation as a percentage of the control value. Values represent the mean \pm standard error of the mean (SEM) $(n=5$ or 6$)$.

The chemical structure of the amino acids and their respective values of gastric adaptive relaxation are shown in Fig. 4. The shorter alkyl chain amino acids, glycine and L-serine, significantly enhanced gastric adaptive relaxation. On the contrary, the longer alkyl chain amino acids, L-alanine and L-threonine, as compared with glycine and L-serine, respectively, did not enhance gastric adaptive relaxation. Similarly, the branched chain amino acids having longer alkyl chains when compared with glycine and L-serine also did not enhance gastric adaptive relaxation. 


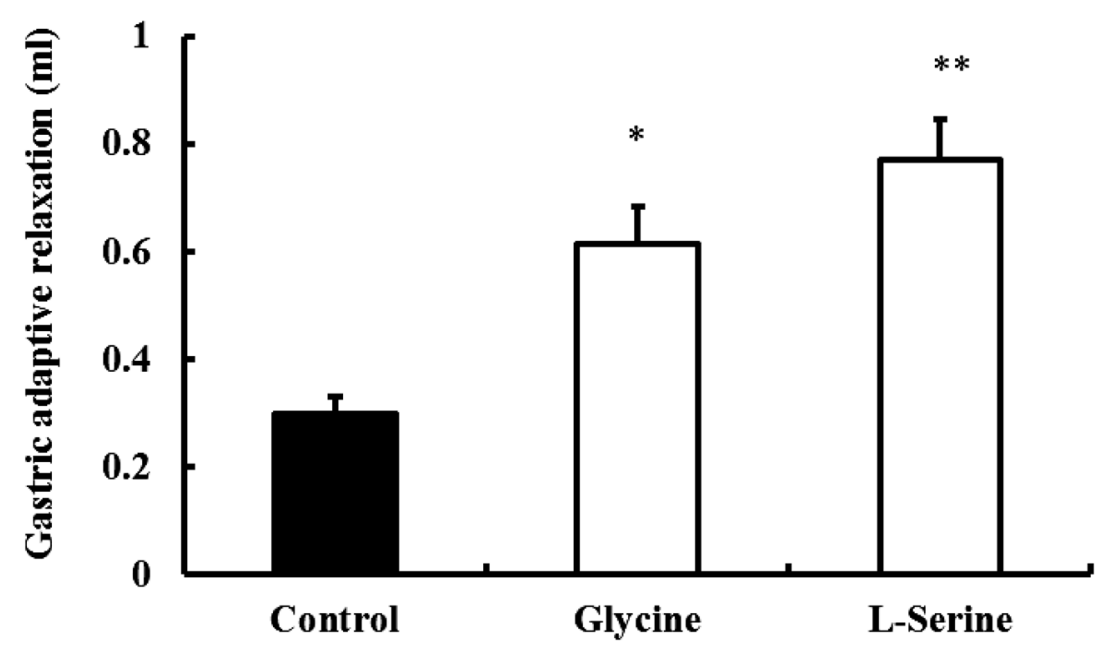

Fig. 5. Effects of intravenously administered glycine and L-serine on gastric adaptive relaxation in rats. In control rats, gastric adaptive relaxation was $0.30 \pm 0.03 \mathrm{ml}$ at $8 \mathrm{mmHg}$. Significant differences were observed following intravenous administration of both glycine and L-serine $(P<0.05$ and $P<0.01$, respectively). Values represent the mean \pm standard error of the mean $(\mathrm{SEM})(\mathrm{n}=4)$.

\section{Effects of intravenously administered glycine and L-serine on the gastric adaptive relaxation}

The effects of intravenously administered glycine and L-serine on gastric adaptive relaxation are shown in Fig. 5. The balloon volume increased gradually and reached a plateau within $3 \mathrm{~min}$. In the control rats, adaptive relaxation was $0.30 \pm 0.03 \mathrm{ml}$. Glycine and L-serine significantly enhanced the gastric adaptive relaxation (Fig. 5).

\section{Discussion}

As compared with the control, glycine and L-serine significantly enhanced the gastric adaptive relaxation when administered both orally and intravenously. This did not occur with the other amino acids used in the present study. These findings clearly demonstrate that the calorific content of amino acids is not involved at all in the enhancement of gastric adaptive relaxation, because all amino acids were administered orally at the same dosage of $1 \mathrm{~g} / \mathrm{kg}$ ( $4 \mathrm{Kcal} / \mathrm{kg}$ ). In addition, it was found that none of the amino acids tested significantly inhibited gastric adaptive relaxation.

Nitric oxide (NO) has been accepted as a mediator of gastric adaptive relaxation. Adaptive relaxation has been known to be mediated by capsaicin sensitive afferent nerves. Lee et al. (10) reported that acute administration of capsaicin decreased proximal gastric tone and inhibited phasic contractility of the proximal stomach in humans. We have also reported that NO plays an important role in the gastric adaptive relaxation in the rat stomach as reported previously using a barostat (9). Tonini et al. (11) also reported a role for NO- and vasoactive intestinal peptide-containing neurones in the relaxation of human gastric fundus strips. Desai et al. (5) reported that adaptive relaxation in the isolated stomach of guinea pigs is mediated by a nonadrenergic and noncholinergic neurotransmitter substance indistinguishable from NO and derived from L-arginine by NO synthase. These findings show that NO may be involved in the enhancement of gastric adaptive relaxation by glycine and L-serine. Pretreatment with the $N^{\omega}$-nitro-L-arginine methyl ester, NO synthase inhibitor, may clarify the mechanism involved in the adaptive relaxation caused by glycine and L-serine in this study. How- 
ever, pretreatment with $N^{\omega}$-nitro-L-arginine methyl ester significantly inhibited the gastric adaptive relaxation as reported by us using our experimental system (9). Thus, further evaluation would be needed to clarify the mechanism involved in producing the effects of glycine and L-serine using another method, such as in vitro studies of preparations of the fundus of the stomach.

Nagahama et al. (12) reported that orally administered glycine was highly effective against acid reflux esophagitis in rats. This finding may show that glycine would enhance gastric adaptive relaxation and inhibit reflux esophagitis. Li et al. (13) found that glycine had a protective effect on cisplatin nephrotoxicity and that this efficacy was inhibited by treatment with $N^{\omega}$-nitro-L-arginine methyl ester, suggesting the involvement of NO. However, Nagahama et al. (12) found that NO was not involved in the efficacy of glycine against acid reflux esophagitis, because the efficacy was not influenced by the prior subcutaneous administration of $N^{\omega}$-nitroL-arginine methyl ester. Therefore, glycine may not enhance gastric adaptive relaxation via a NO pathway.

Glycine has been known to be converted to serine by serine hydroxymethyltransferase (14). Therefore, the enhancement of gastric adaptive relaxation by glycine may in fact be caused by L-serine derived from glycine. In the present study, L-serine also significantly enhanced gastric adaptive relaxation. Mishra et al. (15) reported that L-serine was a potential antihypertensive agent in chronic $N^{\omega}$-nitro-L-arginine methylester-treated and spontaneously hypertensive rats, supporting our relaxant effect of the gastric smooth muscle by L-serine, even though in experimental tissue from different organs. On the contrary, Mishra et al. (15) reported that glycine, the precursor of L-serine, increased blood pressure in chronic $N^{\omega}$-nitro-L-arginine methylester-treated and spontaneously hypertensive rats. In the present study, both L-serine and glycine significantly enhanced the gastric adaptive relaxation. Therefore, glycine may not cause the enhancement of gastric adaptive relaxation as a result of its conversion to L-serine.

Yim et al. (16) reported the expression of a functional glycine receptor chloride channel that attenuates contraction induced by both a tachykinin and acetylcholine in airway smooth muscle. Therefore, the mechanism of enhancement of adaptive relaxation by glycine may be explained by the presence of glycine receptor chloride channels. However, further studies are needed to clarify the mechanisms involved in the enhancement of gastric adaptive relaxation by glycine or L-serine.

With regard to the correlation between the chemical structures and gastric adaptive relaxation, it would appear that the shortest alkyl chain amino acids, glycine and L-serine, enhance gastric adaptive relaxation, as the longer alkyl chain amino acids, L-alanine, L-threonine, L-leucine, L-isoleucine and L-valine, did not show significant effects on the gastric adaptive relaxation (Fig. 4). Therefore, a short alkyl chain may be effective on the enhancement of gastric adaptive relaxation. However, we reported that L-tryptophan significantly enhanced gastric adaptive relaxation (3). L-tryptophan has an aromatic ring, but not an alkyl chain. To elucidate the speculation that a molecule with a short alkyl chain may be effective in the enhancement of gastric adaptive relaxation, many other compounds having a longer alkyl chain would need to be evaluated for their capacity to produce gastric adaptive relaxation.

Sanaka et al. (17) reported that delayed gastric emptying may cause the gastric adaptive relaxation which occurs with proton pump inhibitor therapy, suggesting that the enhancement of gastric adaptive relaxation induces the delay of gastric emptying. In addition, we also found that L-tryptophan may inhibit gastric emptying through the enhancement of gastric adaptive relaxation (3). These reports suggest that inhibition of gastric emptying may relate to the enhancement of gastric adaptive relaxation. However, a correlation between gastric emptying and gastric adaptive relaxation has not been clarified for amino acids other than L-tryptophan as reported by us (3).

We have previously evaluated the effects of these same amino acids on gastric emptying with the breath 


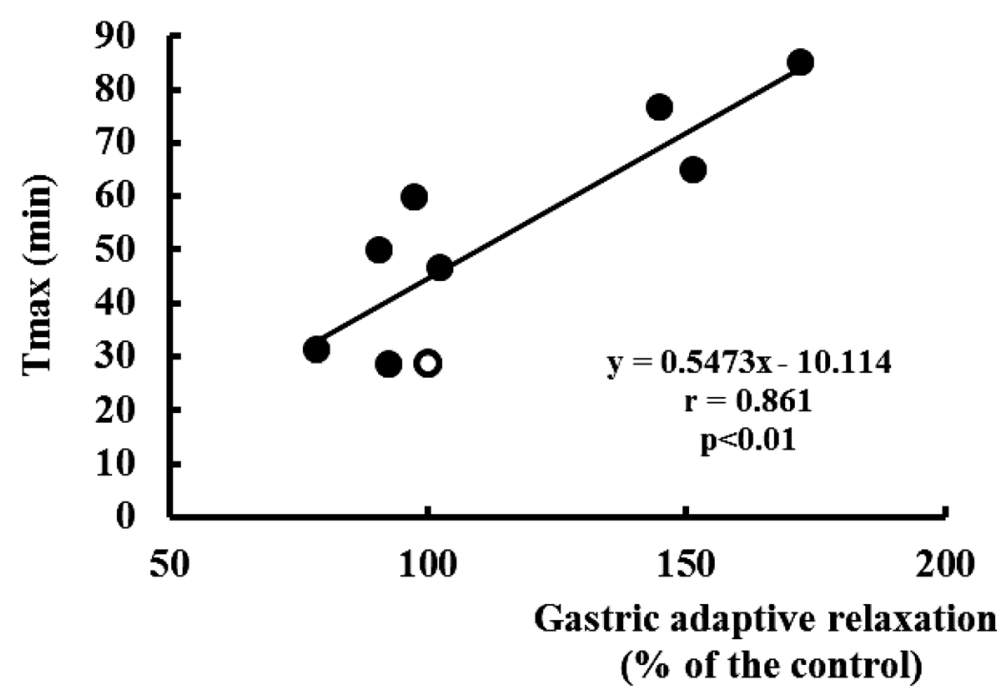

Fig. 6. Correlation in rats between gastric adaptive relaxation and Tmax, one of the parameters for gastric emptying. By adding the data of Tmax value and gastric adaptive relaxation cited from our previous studies to the present results, a significant positive correlation was obtained between Tmax values and gastric adaptive relaxation $(P<0.01)$. Open circle represents control values.

test using $\left[1-{ }^{13} \mathrm{C}\right]$ acetic acid, and found that L-serine significantly delayed gastric emptying. The Tmax and Cmax values were significantly delayed and decreased, respectively, as compared with control, but the AUC$120 \min$ value was not. In addition, glycine also significantly delayed and inhibited gastric emptying, with a significant decrease in the $\mathrm{Cmax}$ and $\mathrm{AUC}_{120 \min }$ as well as a significant delay in the Tmax as compared with the control (4). Then, we analyzed the correlation between gastric adaptive relaxation and gastric emptying. By adding the data for the Tmax values and the gastric adaptive relaxation cited from these previous studies to the present results, a significant positive correlation between the gastric adaptive relaxation and Tmax values was found as shown in Fig. $6(r=0.861, P<0.01)$. However, the other pharmacokinetic parameters, Cmax and $\mathrm{AUC}_{120 \mathrm{~min}}$ values did not show a significant correlation with gastric adaptive relaxation (data not shown). These findings may show that enhancement of gastric adaptive relaxation delays gastric emptying, although these amino acids might have a direct effect on the function of the antrum.

In conclusion, the present results show that glycine and L-serine significantly enhanced gastric adaptive relaxation and that the other amino acids used in the present study did not significantly influence it. Therefore, glycine and L-serine may become useful in the therapy of functional dyspepsia, especially for early satiety, because one of the causes of early satiety is dysfunction of gastric adaptive relaxation. Moreover, it was found that there was a positive correlation between the enhanced gastric adaptive relaxation and the inhibition of gastric emptying as evaluated by the breath test, suggesting that enhanced gastric adaptive relaxation delays gastric emptying.

\section{Conflict of interest}

The authors declare that they have no conflict of interest. 


\section{References}

1. Jordi J, Herzog B, Camargo SM, Boyle CN, Lutz TA, Verrey F. Specific amino acids inhibit food intake via the area postrema or vagal afferents. J Physiol. 2013; 591(22): 5611-21.

2. Carney BI, Jones KL, Horowitz M, Sun WM, Hebbard G, Edelbroek MA. Stereospecific effects of tryptophan on gastric emptying and hunger in humans. J Gastroenterol Hepatol. 1994; 9(6): 557-63.

3. Uchida M, Kobayashi O, Iwamoto C. Effects of L-tryptophan on gastric emptying evaluated by breath test in relation to gastric accommodation evaluated by Barostat in rats. J Pharmacol Sci. 2015; 127: 229-31.

4. Uchida M, Kobayashi O, Iwasawa K, Shimizu K. Effects of straight alkyl chain, extra hydroxylated alkyl chain and branched chain amino acids on gastric emptying evaluated using a non-invasive breath test in conscious rats. J Smooth Muscle Res. 2016; 52: 36-44.

5. Desai KM, Sessa WC, Vane JR. Involvement of nitric oxide in the reflex relaxation of the stomach to accommodate food or fluid. Nature. 1991; 351: 477-9.

6. Desai KM, Zembowicz A, Sessa WC, Vane JR. Nitroxergic nerves mediate vagally induced relaxation in the isolated stomach of the guinea pig. Proc Natl Acad Sci USA. 1991; 88: 11490-4.

7. Ozaki N, Bielefeldt K, Sengupta JN, Gebhart GF. Models of gastric hyperalgesia in the rat. Am J Physiol. 2002; 283: G666-G76.

8. Monroe MJ, Hornby PJ, Partosoedarso ER. Central vagal stimulation evokes gastric volume changes in mice: a novel technique using a miniaturized barostat. Neurogastroenterol Motil. 2004; 16(1): 5-11.

9. Uchida M, Shimizu K. Evaluation of adaptive relaxation of the rat stomach using an orally inserted balloon instead of surgical intervention by demonstrating the effects of capsaicin and $N^{\omega}$-nitro-L-arginine methylester. J Smooth Muscle Res. 2012; 48(4): 97-104.

10. Lee KJ, Vos R, Tack J. Effects of capsaicin on the sensorimotor function of the proximal stomach in humans. Aliment Pharmacol Ther. 2004; 19(4): 415-425.

11. Tonini M, De Giorgio R, De Ponti F, Sternini C, Spelta V, Dionigi P, Barbara G, Stanghellini V, Corinaldesi R. Role of nitric oxide- and vasoactive intestinal polypeptide-containing neurones in human gastric fundus strip relaxations. Br J Pharmacol. 2000; 129(1): 12-20.

12. Nagahama K, Nishio H, Yamato M, Takeuchi K. Orally administered L-arginine and glycine are highly effective against acid reflux esophagitis in rats. Med Sci Monit. 2012; 18(1): BR9-15.

13. Li Q, Bowmer CJ, Yates MS. The protective effect of glycine in cisplatin nephrotoxicity: inhibition with $\mathrm{N}^{\mathrm{G}}$-nitro-L-arginine methyl ester. J Pharm Pharmacol. 1994; 46(5): 346-51.

14. Appaji Rao N, Ambili M, Jala VR, Subramanya HS, Savithri HS. Structure-function relationship in serine hydroxymethyltransferase. Biochim Biophys Acta. 2003; 1647(1-2): 24-9.

15. Mishra RC, Tripathy S, Quest D, Desai KM, Akhtar J, Dattani ID, Gopalakrishnan V. L-Serine lowers while glycine increases blood pressure in chronic L-NAME-treated and spontaneously hypertensive rats. J Hypertens. 2008; 26(12): 2339-48.

16. Yim PD, Gallos G, Xu D, Zhang Y, Emala CW. Novel expression of a functional glycine receptor chloride channel that attenuates contraction in airway smooth muscle. FASEB J. 2011; 25(5): 1706-17.

17. Sanaka M, Yamamoto T, Kuyama Y. Effects of proton pump inhibitors on gastric emptying: a systematic review. Dig Dis Sci. 2010; 55: 2431-40. 